\title{
Similarity Solution for Unsteady MHD Flow Near a Stagnation Point of a Three-Dimensional Porous Body with Heat and Mass Transfer, Heat Generation/Absorption and Chemical Reaction
}

\author{
A.J. Chamkha ${ }^{\dagger 1}$ and S.E. Ahmed ${ }^{2}$

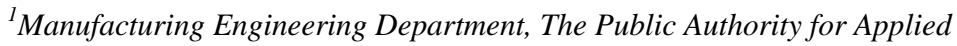 \\ Education and Training, Shuweikh 70654, Kuwait \\ ${ }^{2}$ Department of Mathematics, South Valley University, Qena, Egypt \\ †Corresponding Author Email: achamkha@yahoo.com
}

(Received October 25, 2009; accepted December 30, 2009)

\begin{abstract}
The problem of unsteady mixed convection heat and mass transfer near the stagnation point of a three-dimensional porous body in the presence of magnetic field, chemical reaction and heat source or sink is analyzed. An efficient, iterative, tri-diagonal implicit finite difference method is used to solve the transformed similarity equations in the boundary layer. Three cases were considered, namely, accelerating flow, decelerating flow and the steady-state case. The obtained results are presented in graphical and tabulated forms to illustrate the influence of the different physical parameters such as the magnetic field parameter, transpiration parameter, unsteadiness parameter, ratio of velocity gradients at the edge of the boundary layer parameter, heat generation/absorption parameter and the chemical reaction parameter on the velocity components in the $\mathrm{x}$-and $\mathrm{y}$ - directions, temperature and concentration distributions, as well as the skin-friction coefficients and Nusselt and Sherwood numbers.
\end{abstract}

Keywords: Mixed convection, Three dimensional flow, Stagnation point; Heat source/sink.

\section{NOMENCLATURE}

$\begin{array}{cl}\mathrm{a}, \mathrm{b} & \begin{array}{l}\text { velocity gradient parameters at the boun } \\ \text { dary layer edge in the x- and y-directions } \\ \text { ratio of the velocity gradients at the edge } \\ \text { of the boundary layer } \\ \text { specific heat of the fluid }\end{array} \\ C_{p} & \begin{array}{l}\text { mass diffusion coefficient } \\ \text { gravitational acceleration }\end{array} \\ \mathrm{D} & \text { dimensionless stream functions } \\ \mathrm{f} & \text { fluid thermal conductivity } \\ \mathrm{k} & \text { chemical reaction parameter } \\ k_{c} & \text { magnetic field parameter } \\ \mathrm{M} & \text { local Nusselt number } \\ N u_{x} & \text { Prandtl number } \\ \mathrm{Pr} & \text { heat generation/absorption coefficient } \\ Q_{0} & \text { Reynolds number } \\ \mathrm{Re}_{x} & \text { dimensionless stream function } \\ \mathrm{S} & \text { Schmidt number } \\ \mathrm{Sc} & \text { local Sherwood number } \\ S h_{x} & \text { dimensional temperature } \\ \mathrm{T} & \text { dimensional time }\end{array}$

\begin{tabular}{|c|c|}
\hline$u_{e}$ & dimensional free stream velocity compo \\
\hline$v$ & $\begin{array}{l}\text { nent in } x \text {-direction. } \\
\text { dimensional velocity component in y- } \\
\text { direction }\end{array}$ \\
\hline$v_{e}$ & dimensional free stream velocity compo \\
\hline$w$ & $\begin{array}{l}\text { nent in y-direction } \\
\text { dimensional velocity component in z- } \\
\text { direction }\end{array}$ \\
\hline $\mathrm{x}, \mathrm{y}, \mathrm{z}$ & $\begin{array}{l}\text { longitudinal, transverse and normal } \\
\text { directions }\end{array}$ \\
\hline Greeks & \\
\hline$\beta$ & thermal expansion coefficient \\
\hline$\beta_{c}$ & compositional expansion coefficient \\
\hline$\beta_{0}$ & magnetic induction \\
\hline$\eta$ & similarity variable \\
\hline$v$ & kinematic viscosity \\
\hline$\varphi$ & dimensionless concentration \\
\hline$\rho$ & density of the fluid \\
\hline$\sigma$ & electrical conductivity \\
\hline$\tau$ & dimensionless time parameter \\
\hline
\end{tabular}


A.J. Chamkha and S.E. Ahmed / JAFM, Vol. 4, No. 2, Issue 1, pp. 87-94, 2011.

$\mathrm{u} \quad$ dimensional velocity component in $\mathrm{x}-$ direction.

$\theta \quad$ dimensionless temperature

\author{
Subscripts \\ $w \quad$ condition at the wall \\ $\infty \quad$ ambient condition
}

\section{INTRODUCTION}

Hydromagnetic incompressible viscous flow has many important engineering applications such as magnetohydrodynamic power generators and the cooling of reactors. Chakrabarti and Gupta (1979) studied the MHD flow of Newtonian fluids initially at rest, over a stretching sheet at a different uniform temperature. Vajravelu and Hadjinicolaou (1997) made analysis to flows and heat transfer characteristics in an electrically- conducting fluid near an isothermal sheet. Liao (2006) obtained an accurate series solution of unsteady boundary layer flows over an impulsively stretching plate uniformly valid for all non-dimensional times. Cheng and Huang (2004) considered the problem of unsteady flows and heat transfer in the laminar boundary layer on a linearly accelerating surface with suction or blowing in the absence and presence of a heat source or sink. Xu and Liao (2005) investigated unsteady MHD flow of a non-Newtonian fluid over a non-impulsively stretching flat sheet and presented an accurate series solution. The problem of magnetohydrodynamic natural convection about a vertical impermeable flat plate is presented by Sparrow and Cess (1961) and Wilks and Hunt (1984). Watanabe and Pop (1993) studied the case of a wedge. The laminar plume above a line heat source in a transverse magnetic field was studied by Gray (1997). Kafoussias (1992) investigated the MHD free convective flow through a porous medium over an isothermal cone surface. Takhar et al. (1986) studied MHD symmetric flow past a semi infinite moving plate and reported numerical solutions. Yih (1999) studied free convection effects on MHD coupled heat and mass transfer of a moving permeable vertical surface.

On the other hand, the study of heat generation or absorption in moving fluids is important in problems dealing with chemical reactions and those concerned with dissociating fluids. Possible heat generation effects may alter the temperature distribution and consequently, the particle deposition rate in nuclear reactors, electronic chip sand semi-conductor wafers. In fact, the literature is replete with examples dealing with the heat transfer in laminar flow of viscous fluids. Similarity solution of natural convection boundary layers adjacent to vertical and horizontal surfaces in porous media with internal heat generation was studied by Pop and Postelnicu (1999). Chamkha (2003) studied the problem of MHD flow of a uniformally stretched vertical permeable surface in the presence of heat generation/absorption and chemical reaction. Unsteady heat and mass transfer from a rotating vertical cone with a magnetic field and heat generation or absorption effects was studied by Chamkha and Al-Mudhaf (2005). Chamkha et al. (2006) presented analysis of the effect of heat generation or absorption on thermophoretic free convection boundary layer from a vertical flat plate embedded in a porous medium. Bararnia et al. (2009) investigated analytically the problem of MHD natural convection flow of a heat generation fluid in a porous medium. The effects of mass transfer on flow past an impulsively started infinite vertical plate with constant heat flux and chemical reaction were studied by Das et al. (1994). Andersson et al. (1994) studied the flow and mass diffusion of a chemical species with first-order and higher order reactions over a linearly stretching surface. Sharma and Singh (2009) presented a numerical solution for the problem of effects of variable thermal conductivity and heat source / sink on MHD flow near a stagnation point on a linearly stretching sheet.

Moreover, three-dimensional (3D) mixed convection boundary-layer flows appear to have received (to the authors' knowledge) relatively little attention in the literature in comparison with their $2 \mathrm{D}$ counterparts so far ; yet in most engineering practices flows are more likely to be rather 3D in nature than not. The difficulty encountered in studying such flows may somewhat be alleviated in considering examples tenable to analyses with a view to providing a deeper insight into the more complex flow situations. In this spirit, Yao and Catton (1977) considered the laminar boundary-layer over a heated hollow semi-infinite cylinder with its axis aligned parallel to a uniform stream and normal to the direction of gravity. An interesting example of 3D mixed convection is furnished by the work of Eichhorn and Hasan (1980) where the buoyancy force acts in a direction perpendicular to that of a free stream flowing past a wedge. Unsteady mixed convection flow at a three-dimensional stagnation point with the effect of large injection rates was analyzed by Eswara and Nath (1999).

The main objective of this paper is to study the effects of heat generation/absorption and chemical reaction on unsteady MHD flow heat and mass transfer near a stagnation point of a three dimensional porous body in the presence of a uniform magnetic field. An efficient, iterative, tri-diagonal implicit finite difference method is used to solve the transformed similarity equations in the boundary layer.

\section{GOVERNING EQUATIONS}

Consider unsteady laminar incompressible boundary layer flow of a viscous electrically-conducting fluid at a three-dimensional stagnation point with magnetic field, chemical reaction, heat generation/absorption and suction/injection effects. A uniform transverse magnetic field is assumed to be applied normal to the body surface. The fluid properties are assumed to be constant and the chemical reaction is taking place in the flow. The velocity components of the inviscid flow over the three dimensional body surface are given by:

$$
u_{e}(x, t)=\operatorname{ax\varphi }(\tau), \quad v_{e}(x, t)=\operatorname{by} \varphi(\tau)
$$


It is assumed that near the stagnation point, the free stream temperature is constant and that viscous dissipation effects are neglected. Notice that for $T_{w}>T_{\infty}$ the buoyancy force, which arises due to temperature differences, will aid the forced flow. On the other hand, for $T_{w}<T_{\infty}$, the resulting buoyancy force will oppose the forced flow. Under these assumptions as well as Boussinesq approximation, the continuity, momentum, energy and concentration equations are given by

$\frac{\partial u}{\partial x}+\frac{\partial v}{\partial y}+\frac{\partial w}{\partial z}=0$

$\frac{\partial u}{\partial t}+u \frac{\partial u}{\partial x}+v \frac{\partial u}{\partial y}+w \frac{\partial u}{\partial z}=\frac{\partial u_{e}}{\partial t}+u_{e} \frac{\partial u_{e}}{\partial x}+v \frac{\partial^{2} u}{\partial z^{2}}-\frac{\sigma \beta_{0}^{2}}{\rho}\left(u-u_{e}\right)$

$+\left[g \beta\left(T-T_{\infty}\right)+g \beta_{c}\left(C-C_{\infty}\right)\right] x / l$,

$\frac{\partial v}{\partial t}+u \frac{\partial v}{\partial x}+v \frac{\partial v}{\partial y}+w \frac{\partial v}{\partial z}=\frac{\partial v_{e}}{\partial t}+v_{e} \frac{\partial v_{e}}{\partial y}+v \frac{\partial^{2} v}{\partial z^{2}}-\frac{\sigma \beta_{0}^{2}}{\rho}\left(v-v_{e}\right)$ $+\left[g \beta\left(T-T_{\infty}\right)+g \beta_{c}\left(C-C_{\infty}\right)\right] c y / l$,

$\frac{\partial T}{\partial t}+u \frac{\partial T}{\partial x}+v \frac{\partial T}{\partial y}+w \frac{\partial T}{\partial z}=\frac{k}{\rho C_{p}} \frac{\partial^{2} T}{\partial z^{2}}+\frac{Q_{0}}{\rho C_{p}}\left(T-T_{\infty}\right)$,

$\frac{\partial C}{\partial t}+u \frac{\partial C}{\partial x}+v \frac{\partial C}{\partial y}+w \frac{\partial C}{\partial z}=D \frac{\partial^{2} C}{\partial y^{2}}-k_{c}\left(C-C_{\infty}\right)$.

The appropriate initial and boundary conditions are

$$
\begin{aligned}
t=0: u(x, y, z, t) & =u_{i}(x, y, z), v(x, y, z, t)=v_{i}(x, y, z), \\
w(x, y, z, t) & =w_{i}(x, y, z), T(x, y, z, t)=T_{i}(x, y, z), \\
C(x, y, z, t) & =C_{i}(x, y, z), \\
t>0: u(x, y, z, t) & =0, v(x, y, z, t)=0, w(x, y, z, t)=w_{w}, \\
T(x, y, z, t) & =T_{w}, C(x, y, z, t)=C_{w} \text { at } y=0, \\
t>0: u(x, y, z, t) & =u_{e}(x, y), v(x, y, z, t)=v_{e}(x, y), \\
T(x, y, z, t) & =T_{\infty}, C(x, y, z, t)=C_{\infty} \text { as } y \rightarrow \infty .
\end{aligned}
$$

\section{SIMILARITY ANALYSIS}

It is possible to transform Eqs.(3)-(6) into a set of selfsimilar equations by assuming that the free stream velocity vary inversely as a linear function of time. Substituting the following dimensionless quantities

$\eta=z\left(\frac{a}{v(1-\lambda \tau}\right)^{1 / 2}, \tau=a t, u=a x(1-\lambda \tau)^{-1} f(\eta)$,

$v=b y(1-\lambda \tau)^{-1} s(\eta), \lambda \tau<1, w=-\sqrt{a v}(1-\lambda \tau)^{-1 / 2}(f+c s)$,

$\theta(\eta)=\frac{T-T_{\infty}}{T_{w}-T_{\infty}}, \Phi(\eta)=\frac{C-C_{\infty}}{C_{w}-C_{\infty}}, c=b / a$

$\overline{\operatorname{Re}_{x}}=a x^{2} /[v(1-\lambda \tau)], M=\sigma \beta_{0}^{2} v \overline{\operatorname{Re}_{x}} / \rho x^{2} a^{2}$,

$\delta=Q_{0} v \overline{\mathrm{Re}_{x}} / \rho C_{p} x^{2} a^{2}, \gamma=k_{c} v \overline{\mathrm{Re}_{x}} / x^{2} a^{2}$,

$f_{w}=\sqrt{a v(1-\lambda \tau)} w_{w}$

into Eqs. (3)-(6) yields the following similarity equations:

$$
\begin{aligned}
& f^{\prime \prime \prime}+f^{\prime \prime}(f+c s-\lambda \eta / 2)-f^{\prime}\left(\lambda+f^{\prime}\right) \\
& +(\lambda+1)+M\left(f^{\prime}-1\right)+\lambda_{1} \theta+\lambda_{2} \Phi=0, \\
& s^{\prime \prime \prime}+s^{\prime \prime}(f+c s-\lambda \eta / 2)-s^{\prime}\left(\lambda+c s^{\prime}\right) \\
& +(\lambda+c)+M\left(s^{\prime}-1\right)+\lambda_{1} \theta+\lambda_{2} \Phi=0, \\
& \frac{1}{\operatorname{Pr}} \theta^{\prime \prime}+\theta^{\prime}(f+c s-\lambda \eta / 2)+\delta \theta=0, \\
& \frac{1}{S c} \Phi^{\prime \prime}+\Phi^{\prime}(f+c s-\lambda \eta / 2)-\gamma \Phi=0 .
\end{aligned}
$$

where $\operatorname{Pr}=\mu C_{p} / k$ is the Prandtl number, $G r=g \beta L^{3}\left(T_{w}-T_{\infty}\right) / v^{2}$ is the Grashof number, $G r_{C}=g \beta_{c} L^{3}\left(C_{w}-C_{\infty}\right) / v^{2}$ is the modified Grashof number, $\operatorname{Re}_{L}=a L^{2} / v$ is Reynolds number, $\left(\lambda_{1}=G r / \operatorname{Re}_{L}^{2}, \lambda_{2}=G r_{C} / \operatorname{Re}_{L}^{2}\right)$ are the buoyancy parameters, $S c=v / D$ is the Schmidt number, $M=\sigma \beta_{0}^{2} / \rho a$ is the magnetic field parameter, $\delta=Q_{0} / \rho C_{p} a$ is the heat generation/absorption parameter and $\gamma=k_{c} / a$ is the chemical reaction parameter. $\lambda$ denotes the unsteadiness parameter and the range of $\lambda$ in this investigation is $-1<\lambda<1$. The case $\lambda>0$ represents the accelerating flow case provided $\lambda \tau<1$ and the case $\lambda<0$ corresponds to a decelerating flow. However, the steady-state case can be obtained by setting $\lambda=0$.

In addition, the transformed boundary conditions (7) become

$\eta=0: f^{\prime}=s^{\prime}=0, f=-f_{w}, \theta=1, \quad \Phi=1$,

$\eta \rightarrow \infty: f^{\prime}=s^{\prime}=1, \theta=0, \quad \Phi=0$.

In the above equation, $f_{w}=\left(w_{w} / u_{e}\right) \sqrt{\operatorname{Re}_{x}}$ is the suction/injection parameter and $f_{w}>0$ or $f_{w}<0$ according to whether there is a suction or injection, respectively.

The coefficients of the local skin friction, local Nusselt number and the local Sherwood number are given, respectively by

$C_{f x}=\frac{2 \mu\left[\frac{\partial u}{\partial z}\right]_{w}}{\rho\left(u_{e}\right)^{2}}$

$C_{f y}=\frac{2 \mu\left[\frac{\partial v}{\partial z}\right]_{w}}{\rho\left(u_{e}\right)^{2}}$,

$N u_{x}=\frac{-x\left[\frac{\partial T}{\partial z}\right]_{w}}{T_{w}-T_{\infty}}$,

$S h_{x}=\frac{-x\left[\frac{\partial C}{\partial z}\right]_{w}}{C_{w}-C_{\infty}}$.

By using Eq. (8), we can get 


$$
\begin{aligned}
& C_{f x}\left(\operatorname{Re}_{x}\right)^{1 / 2}=2 \varphi(\tau) f^{\prime \prime}(0), \\
& C_{f y}\left(\operatorname{Re}_{x}\right)^{1 / 2}=2\left(u_{e} / v_{e}\right) \varphi(\tau) s^{\prime \prime}(0), \\
& \left(\operatorname{Re}_{x}\right)^{-1 / 2} N u_{x}=-\theta^{\prime}(0), \\
& \left(\operatorname{Re}_{x}\right)^{-1 / 2} S h_{x}=-\Phi^{\prime}(0) .
\end{aligned}
$$

\section{RESULTS AND DISCUSSION}

In order to get a clear insight of the physical problem, numerical results are displayed with the help of graphical and tabulated illustrations. The system of Eqs. (9)-(12) with the boundary conditions (13) were solved numerically by means of an efficient, iterative, tridiagonal implicit finite-difference method discussed previously by Blottner (1970).

Equations (9)-(12) are discretized using three-point central difference formulae with $f^{\prime}$ replaced by another variable $\mathrm{V}$. The $\eta$ direction is divided into 196 nodal points and a variable step size is used to account for the sharp changes in the variables in the region close to the surface where viscous effects dominate. The initial step size used is $\Delta \eta_{1}=0.001$ and the growth factor $K=1.037$ such that $\Delta \eta_{n}=K \Delta \eta_{n-1}$ (where the subscript $\mathrm{n}$ is the number of nodes). This gives $\eta_{\max } \approx 35$ which represents the edge of the boundary layer at infinity. The ordinary differential equations are then converted into linear algebraic equations that are solved by the Thomas algorithm discussed by Blottner (1970). Iteration is employed to deal with the nonlinear nature of the governing equations. The convergence criterion employed in this work was based on the relative difference between the current and the previous iterations. When this difference or absolute error reached $10^{-5}$, then the solution was assumed converged and the iteration process was terminated.

It is possible to compare the results obtained by this numerical method with the previously published work of Eswara and Nath (1999). Table 1 shows that a good agreement between the results exists. This lends confidence in the numerical results to be reported subsequently.

Table 1 Comparison between our results and Eswara and Nath (1999) when $\lambda_{1}=3.0, c=0.5, \operatorname{Pr}=0.7$

\begin{tabular}{ccccc}
\hline & \multicolumn{2}{c}{ Present results } & \multicolumn{2}{c}{$\begin{array}{c}\text { Eswara and Nath } \\
(1999)\end{array}$} \\
\cline { 2 - 5 }$A=0.5$ & $\lambda=-0.5$ & \multicolumn{2}{c}{$\lambda=0.5$} & $\lambda=-0.5$ \\
\hline \multirow{2}{c}{$\lambda$} & \multicolumn{2}{c}{$C_{f x}\left(\operatorname{Re}_{x}\right)^{1 / 2}$} & \multicolumn{2}{c}{$C_{f x}\left(\operatorname{Re}_{x}\right)^{1 / 2}$} \\
3.0 & 3.31212 & 2.59404 & 3.3333 & 2.6111 \\
4.0 & 2.60624 & 2.19012 & 2.6388 & 2.2222 \\
6.0 & 2.11676 & 1.75013 & 2.1666 & 1.7778 \\
8.0 & 1.74914 & 1.37065 & 1.7777 & 1.3888 \\
10 & 1.40791 & 1.10003 & 1.4166 & 1.1111 \\
28 & 0.81096 & 0.57914 & 0.8333 & 0.5833 \\
\hline
\end{tabular}

Computations were carried out for the various values of parameters, the value of Prandtl number $\operatorname{Pr}=0.71$, which corresponds, physically to the air and the value of Schmidt number $S c=0.22$ which represents hydrogen. The results of this parametric study are shown in Figs. 1-16.

Figures 1-4 show the effects of the magnetic field parameter $M$ and the suction/injection parameter $f_{w}$ on the velocity components in $\mathrm{x}$ - and $\mathrm{y}$-directions, temperature distributions and concentration distributions in the case of accelerating flow $\lambda>0$, respectively. The presence of a magnetic field in the flow field creates a drag-like force called the Lorentz force which has the tendency to slow down the flow motion and to increases the fluid temperature and the solute concentration in the fluid. In addition, increasing the value of suction/blowing parameter leads to accelerate the fluid flow and increase its temperature and solute concentration in the fluid.

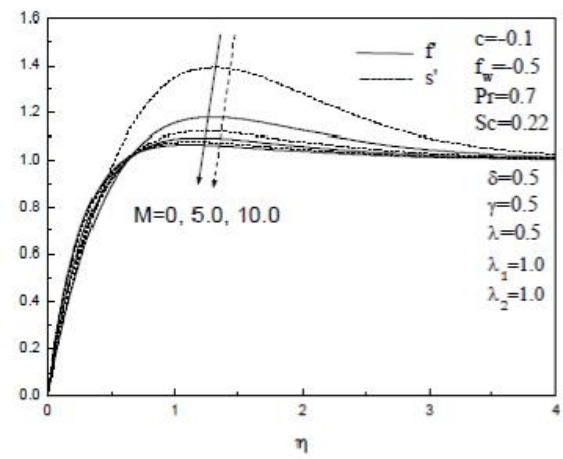

Fig. 1. Effect of magnetic field parameter on velocity components for the case of accelerating flow

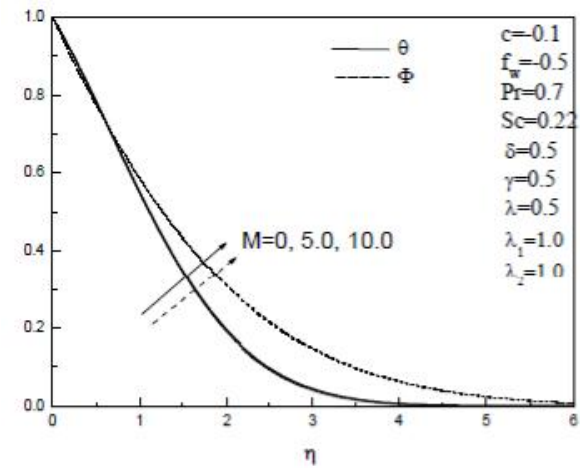

Fig. 2. Effect of magnetic field parameter on temperature and concentration profiles for the case of accelerating flow

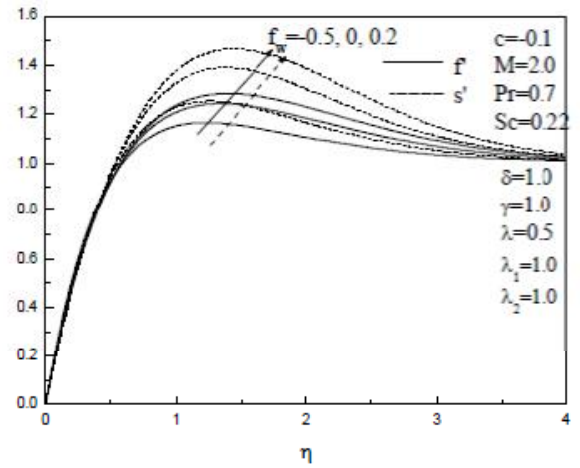

Fig. 3. Effect of transpiration parameter on velocity components for the case of accelerating flow 


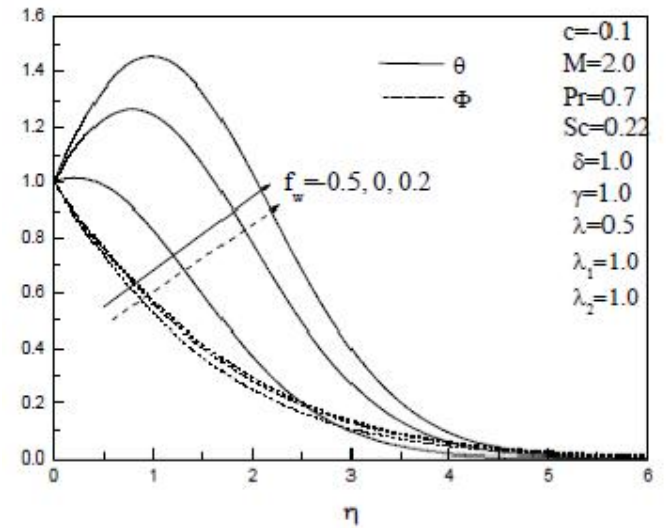

Fig. 4. Effect of transpiration parameter on temperature and concentration profiles for the case of accelerating flow

Figures 5 and 6 show the effects of the magnetic field parameter $M$ on the local skin-friction coefficients $C_{f x}\left(\operatorname{Re}_{x}\right)^{1 / 2}$ and $C_{f y}\left(\operatorname{Re}_{x}\right)^{1 / 2}$, Nusselt number $\left(\operatorname{Re}_{x}\right)^{-1 / 2} N u_{x}$ and the Sherwood number $\left(\operatorname{Re}_{x}\right)^{-1 / 2} S h_{x}$ for different values of the transpiration parameter $f_{w}$ for the case of accelerating flow $(\lambda>0)$, respectively. It is clear that increasing the value of the magnetic field parameter results in increases in the skin-friction coefficients and decreases in both of the Nusselt and Sherwood numbers. In addition, increasing the value of the transpiration parameter results in decreases not only in the skin-friction coefficients but in the Nusselt and Sherwood numbers as well.

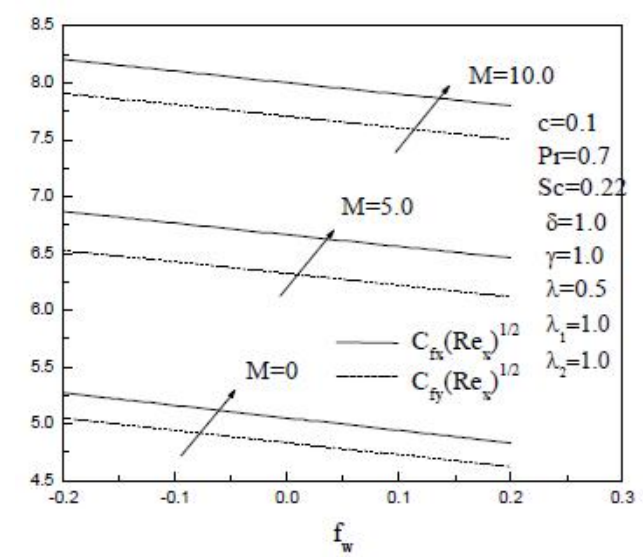

Fig. 5. Effects of the magnetic field parameter $M$ and transpiration parameter $f_{w}$ on the local skin-friction coefficients

With the help of Figs. 7 and 8, the effect of the ratio of the velocity gradients at the edge of the boundary layer $c$ on the velocity components in $\mathrm{x}$ - and $\mathrm{y}$-directions, temperature and concentration profiles for the case of accelerating flow $\lambda>0$ is understood. It is observed that the largest rate of fluid motion can be obtained by considering the smallest values of the ratio of velocity gradients at the edge of the boundary layer. Moreover, the temperature and concentration profiles increase with the decreasing of the ratio of velocity gradients at the edge of the boundary layer.

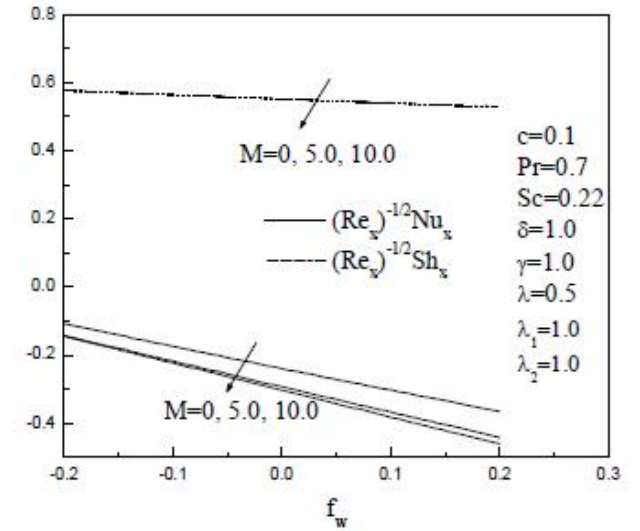

Fig. 6. Effects of the magnetic field parameter $M$ and transpiration parameter $f_{w}$ on the local Nusselt and

Sherwood numbers

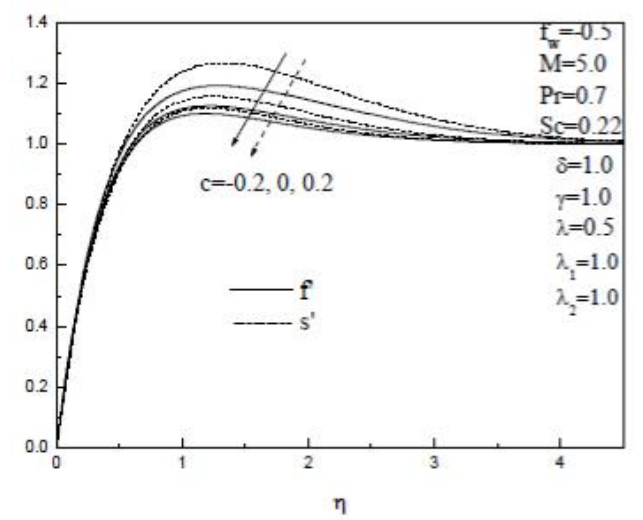

Fig. 7. Effect of ratio of velocity gradients at the edge of the boundary layer on velocity components for the case of accelerating flow

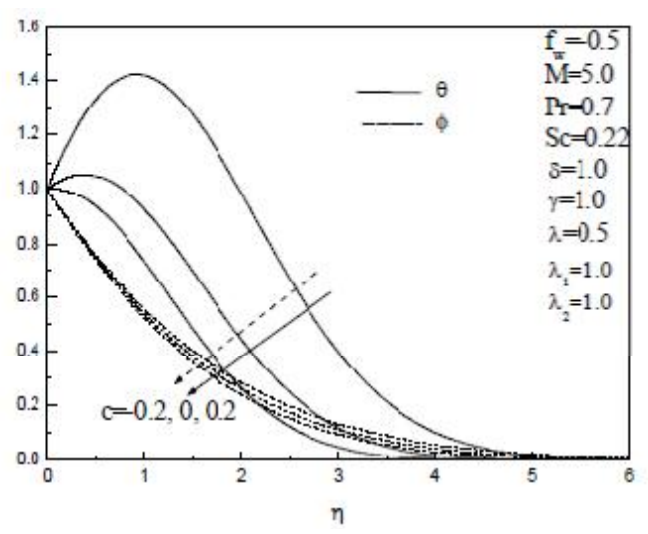

Fig. 8. Effect of ratio of velocity gradients at the edge of the boundary layer on temperature and concentration profiles for the case of accelerating flow

Figures 9 and 10 display the effects of the heat generation/absorption parameter $\delta$ on the velocity components in $\mathrm{x}$ - and $\mathrm{y}$-directions, temperature and the solute concentration in the fluid for the case of decelerating flow $(\lambda<0)$, respectively. In general, the presence of a heat source $\delta>0$ in the flow field caused the fluid temperature to increase. This leads to increase the activity of motion due to increase in the induced buoyancy-induced flow. On the contrary, the presence 
of a heat sink causes reductions in the fluid temperature and therefore, the thermal buoyancy effects. This leads to a decay of the fluid motion. The solute concentration in the fluid decreases slightly as the heat generation/absorption parameter $\delta$ increases.

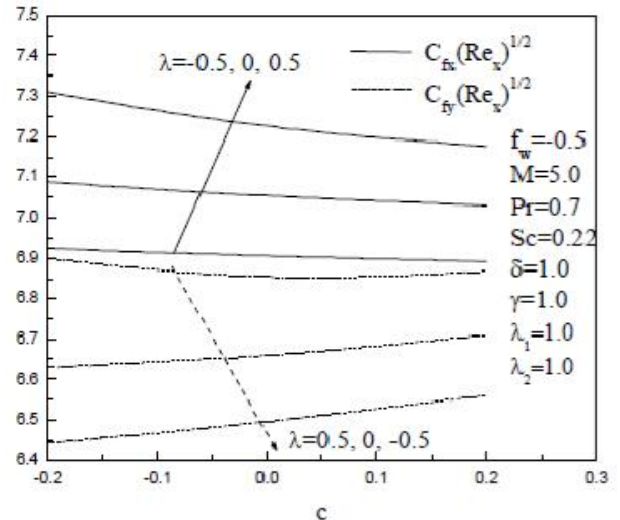

Fig. 9. Effects of the unsteadiness parameter $\lambda$ and ratio of velocity gradients at the edge of the boundary layer $C$ on the local skin-friction coefficients

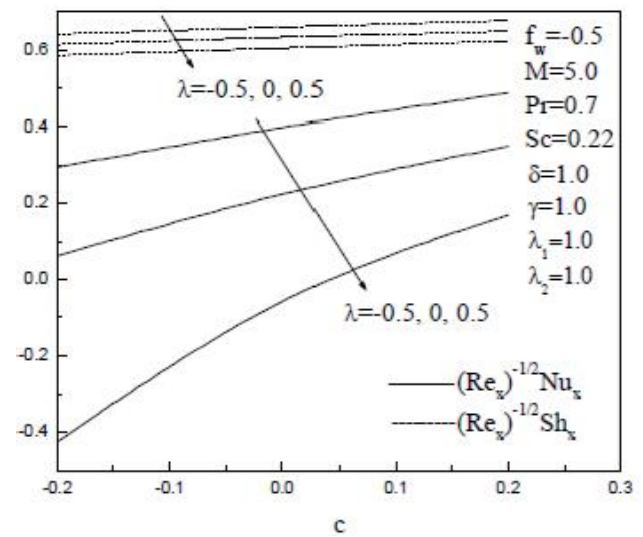

Fig. 10. Effects of the unsteadiness parameter $\lambda$ and ratio of velocity gradients at the edge of the boundary layer $C$ on the local Nusselt and Sherwood numbers

Figures 11 and 12 illustrate the effects of the ratio of velocity gradients at the edge of the boundary layer $C$ on the skin-friction coefficients $C_{f x}\left(\operatorname{Re}_{x}\right)^{1 / 2}$ and $C_{f y}\left(\operatorname{Re}_{x}\right)^{1 / 2}$, Nusselt number $\left(\operatorname{Re}_{x}\right)^{-1 / 2} N u_{x}$ and Sherwood number $\left(\operatorname{Re}_{x}\right)^{-1 / 2} S h_{x}$ for different values of the unsteadiness parameter $\lambda$, respectively. It is observed that as the ratio of velocity gradients at the edge of the boundary layer $c$ increases, the skinfriction coefficient in the y-direction $C_{f y}\left(\operatorname{Re}_{x}\right)^{1 / 2}$, Nusselt number $\left(\operatorname{Re}_{x}\right)^{-1 / 2} N u_{x}$ and the Sherwood number $\left(\operatorname{Re}_{x}\right)^{-1 / 2} S h_{x}$ increase whereas the skin-friction coefficient in the x-direction $C_{f x}\left(\operatorname{Re}_{x}\right)^{1 / 2}$ take the opposite behaviour.

Moreover, as the unsteadiness parameter $\lambda$ increases, the skin-friction coefficients increase whereas both of the Nusselt and Sherwood numbers decrease.
The effects of the chemical reaction parameter $\gamma$ on the on the velocity components in $\mathrm{x}$ - and $\mathrm{y}$-directions, temperature and the solute concentration in the fluid for the steady-state condition $(\lambda=0)$ are shown in Figs. 13 and 14, respectively. Physically, as the chemical reaction parameter $\gamma$ increases, the concentration profiles decrease whereas the temperature of the fluid increases slightly. The increase in solute concentration causes the solutal buoyancy effects to increase resulting in reductions in both of the velocity components. These behaviours are clear from Figs. 13 and 14.

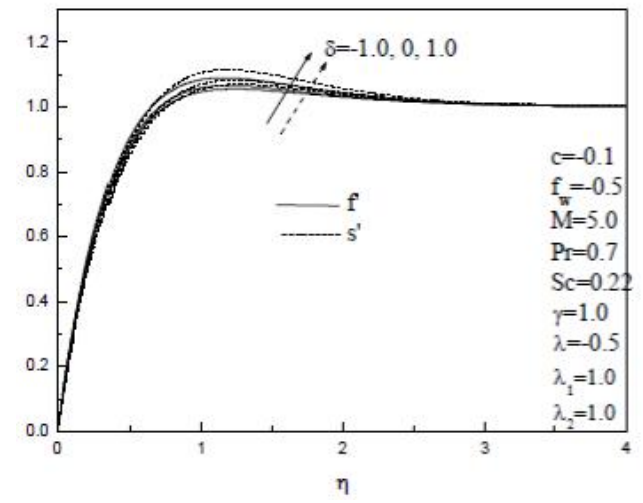

Fig. 11. Effect of heat generation/absorption parameter on velocity components for the case of decelerating flow

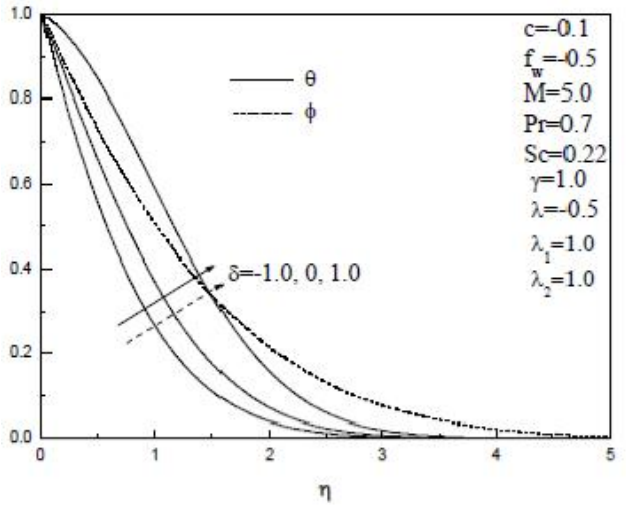

Fig. 12. Effect of heat generation/absorption parameter on temperature and concentration profiles for the case of decelerating flow

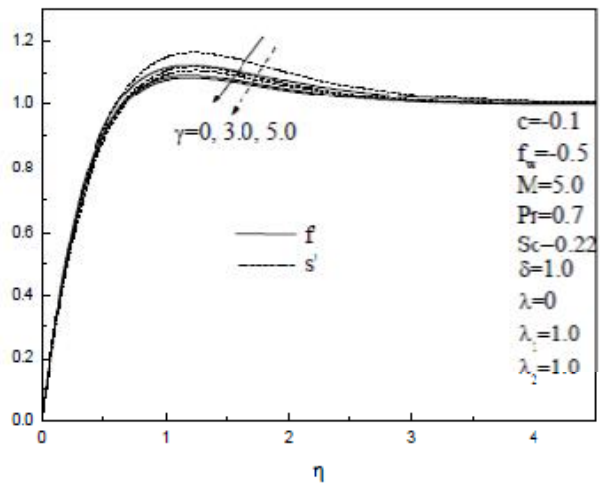

Fig. 13. Effect of chemical reaction parameter on velocity components for the case of steady state flow 


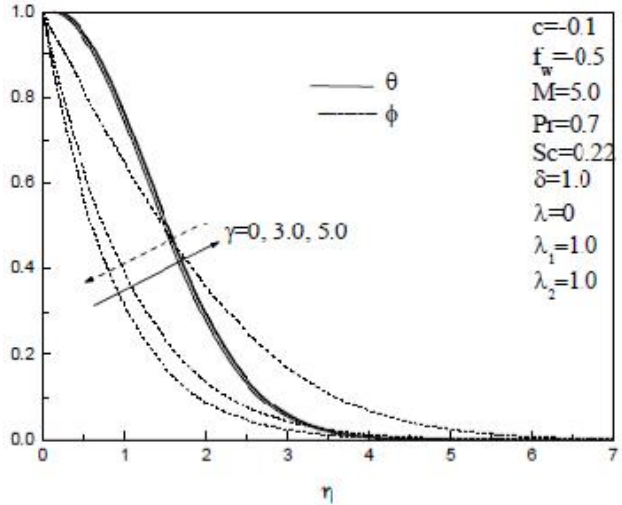

Fig.14. Effect of chemical reaction parameter on temperature and concentration profiles for the case of steady state flow

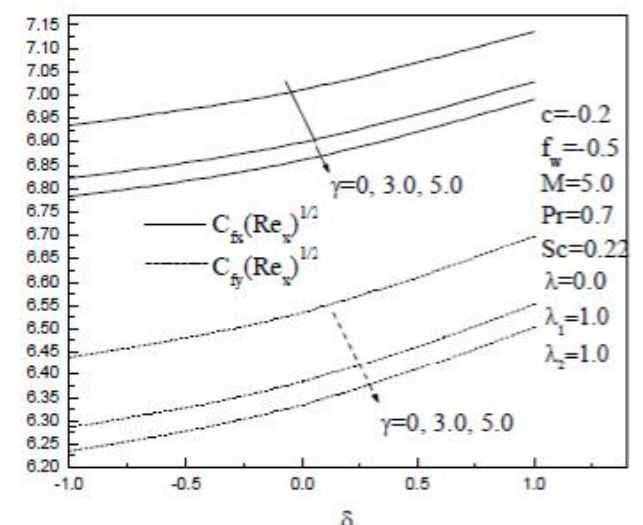

Fig. 15. Effects of the chemical reaction parameters $\gamma$ and the heat generation/absorption parameter $\delta$ on the local skin-friction coefficients

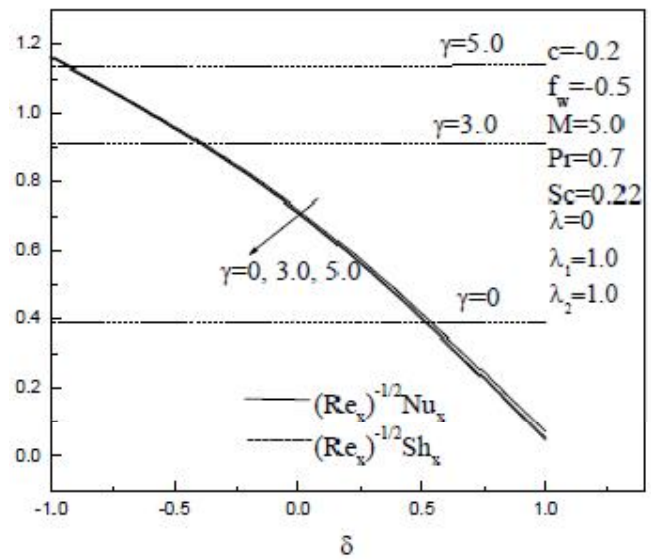

Fig. 16. Effects of the chemical reaction parameters $\gamma$ and the heat generation/absorption parameter $\delta$ on the local Nusselt and Sherwood numbers

Figures 15 and 16 depict the effects of the heat generation/absorption parameter $\delta$ and the chemical reaction parameter $\gamma$ on the skin-friction coefficients $C_{f x}\left(\operatorname{Re}_{x}\right)^{1 / 2}$ and $C_{f y}\left(\operatorname{Re}_{x}\right)^{1 / 2}$, Nusselt number $\left(\operatorname{Re}_{x}\right)^{-1 / 2} N u_{x}$ and the Sherwood number $\left(\operatorname{Re}_{x}\right)^{-1 / 2} S h_{x}$ under steady-state conditions $(\lambda=0)$. It is clearly observed that increasing the value of the heat generation/absorption parameter $\delta$ produces increases in the skin-friction coefficients and the Sherwood number whereas the Nusselt number decreases. In addition, it is predicted that the presence of the chemical reaction $(\gamma>0)$ results in increases in the rates of heat and mass transfer and decreases in the skinfriction coefficients.

\section{Conclusion}

The problem of unsteady MHD mixed convection heat and mass transfer near the stagnation point of a threedimensional porous body in the presence of heat generation/absorption and chemical reaction effects was studied. The governing equations were developed and transformed into a similar form. The self-similar equations were solved numerically by an efficient tridiagonal implicit finite-difference method. From the results of the problem, it is observed that

- Increasing the value of magnetic field parameter resulted in increases in both of the skin-friction coefficients, temperature and solute concentration in the fluid whereas the Nusselt number, Sherwood number and velocity components decreased with the increasing values of the magnetic parameter.

- Imposition of fluid wall suction resulted in decreases in the skin-friction coefficients, Nusselt and Sherwood numbers whereas the velocity components, temperature and concentration increased.

- As expected, the largest rate of the fluid motion was obtained in the case of accelerating flow and the smallest rate of fluid motion was resulted from the decelerating flow case.

- The skin-friction coefficients decreased as either of the chemical reaction parameter or ratio of velocity gradients at the edge of the boundary layer increased and increased as the heat generation/absorption parameter increase.

- The Nusselt number decreased as either of the heat generation/absorption parameter or the chemical reaction parameter increased and increased as the ratio of velocity gradients at the edge of the boundary layer increased.

- The Sherwood number increased as either of the heat generation/absorption parameter, the chemical reaction parameter or the ratio of velocity gradients at the edge of the boundary layer increased.

\section{REFERENCES}

Andersson, K.I., O.R. Hansen, and B. Holmedal (1994). Diffusion of a chemically reactive species from a stretching sheet. International journal of Heat and Mass Transfer 37, 659-664.

Bararnia, H., A.R. Ghotbi and G. Domairry (2009). On the analytical solution for MHD natural convection flow and heat generation fluid in porous medium. Communication in Nonlinear Science and Numerical Simulation 14, 2689-2701. 
Blottner, F.G. (1970). Finite-difference methods of solution of the boundary-layer equations. American Institute of Aeronautics and Astronautics 8, 193-205.

Chakrabarti, A. and A.S. Gupta (1979). Hydromagnetic flow and heat transfer over a stretching sheet. Quarterly Journal of Applied Mathematics 37, 7378 .

Chamkha, A.J. (2003). MHD flow of a uniformally stretched vertical permeable surface in the presence of heat generation/absorption and chemical reaction. International Communication in Heat and Mass Transfer 30, 413-422.

Chamkha, A.J. and A. Al-Mudhaf (2005). Unsteady heat and mass transfer from a rotating vertical cone with a magnetic field and heat generation or absorption effects. International Journal of Thermal Science 44, 267-276.

Chamkha, A.J., A. Al-Mudhaf and I. Pop (2006). Effect of heat generation or absorption on thermophoretic free convection boundary layer from a vertical flat plate embedded in a porous medium. International Communication in Heat and Mass Transfer 33, 1096-1102.

Cheng, W.T. and C.N. Huang (2004). Unsteady flow and heat transfer on an accelerating surface with blowing or suction in the absence and presence of a heat source or sink. Chemical Engineering. Science 59, 771-780.

Das, U.N., R. Deka and V.M. Soundalgekar (1994). Effects of mass transfer on flow past an impulsively started infinite vertical plate with constant heat flux and chemical reaction. Journal of Forschung Im. Ingenieurwesen-Engineering Research Bd. 60, 284-287.

Eichhorn, R. and M. M. Hasan (1980). Mixed convection about a vertical surface in cross-flow: a similarity solution. ASME Journal of Heat Transfer 102, 775-777.

Eswara, A. T. and G. Nath (1999). Effect of large injection rates on unsteady mixed convection flow at a three-dimensional stagnation point. International Journal of Non-Linear Mechanics 34, 85-103.

Gray, D.D.(1977). The laminar plume above a line heat source in a transverse magnetic field. Applied Science Research 33, 437-457.

Kafoussias, N.G. ( 1992). MHD free convective flow through a non homogeneous porous medium over an isothermal cone surface. Mechanics Research Communication 19, 89-99.

Liao, S.J. (2006). An analytic solution of unsteady boundary-layer flows caused by an impulsively stretching plate. Communication in Nonlinear Science and Numerical Simulation 11, 326-339.

Pop, I. and A. Postelnicu (1999). Similarity solutions of free convection boundary layers over vertical and horizontal surface porous media with internal heat generation. International Communication in Heat and Mass Transfer 26 1183-1191.

Sharma, P.R. and G. Singh (2009). Effects of variable thermal conductivity and heat source / sink on MHD flow near a stagnation point on a linearly stretching sheet. Journal of Applied Fluid Mechanics 2, 13-21.

Sparrow, E.M. and R.D. Cess (1961). The effect of a magnetic field on free convection heat transfer. International Journal of Heat and Mass Transfer 4, 267-74.

Takhar, H.S., A.A. Raptis and C.P. Perdikis (1986). MHD a symmetric flow past a semi- infinite moving plate. Acta. Mechanics 65, 287-290.

Vajravelu, K. and A. Hadjinicolaou (1997). Convective heat transfer in an electrically conducting fluid at a stretching surface with uniform free stream. International Journal of Engineering Science 35, 1237-1244.

Watanabe, T. and I. Pop (1993). Magnetohydrodynamic free convection flow over a wedge in the presence of a transverse magnetic field. International Communication in Heat and Mass Transfer 20, 871-881.

Wilks, G. and R. Hunt (1984). Magnetohydrodynamic free convection flow about a semi- infinite plate at whose surface the heat flux is uniform. Journal of Applied Mathematical Physics 35, 34-49.

Xu, H. and S.J. Liao (2005). Analytic solutions of magnetohydrodynamic flows of non- Newtonian fluids caused by an impulsively stretching plate. Journal of Non-Newton Fluid Mechanics 159, 4655.

Yao, L.S. and I. Catton (1977). Buoyancy cross-flow effects on longitudinal boundary layer flow along a heated horizontal hollow cylinder. ASME Journal of Heat Transfer 99, 122- 124.

Yih, K.A. (1999). Free convection effect on MHD coupled heat and mass transfer of a moving permeable vertical surface. International Communication in Heat and Mass Transfer 26, 95-104. 